\title{
Wavelet analysis of light emission signals in laser beam welding
}

\author{
Farzad Vakili-Farahani \\ Laboratory of Advanced Material Processing, Swiss Federal Laboratory for Materials Science \\ and Technology (Empa), Feuerwerkerstrasse 39, CH-3602 Thun, Switzerland \\ Jörn Lungershausen \\ Rofin-Lasag AG, Aemmenmattstrasse 43, CH-3123 Belp, Switzerland \\ Kilian Wasmer \\ Laboratory of Advanced Material Processing, Swiss Federal Laboratory for Materials Science \\ and Technology (Empa), Feuerwerkerstrasse 39, CH-3602 Thun, Switzerland
}

(Received 28 March 2017; accepted for publication 28 March 2017; published 21 June 2017)

\begin{abstract}
This paper introduces a compact, robust, and versatile generation of a laser processing head coupled with a single-mode Ytterbium source StarFiber $150 \mathrm{P}$ which not only is used in a wide range of laser material processing applications but also enables in situ and real-time quality control in production lines. In this study, the effect of power density on the weld quality of bead on plate in a SHADOW (Stepless high speed accurate and discrete one pulse welding) welding process was considered. Furthermore, the co-axial back reflected/emitted light from the process zone was measured using three optical sensors; each one measures the light emission in a certain waveband (450-850, 1000-1200, and 1250-1700 nm). From these sensors, the recorded signals were first used as benchmarks to correlate with the weld quality features and defects. Discrete wavelet transform has been used to analyze the signal within the time-frequency domain. The detail signals at different decomposition levels disclosed information with regard to the type (mode) and dynamics of welding process. Accordingly, the deep penetration welding (keyhole) was confidentially distinguished from the shallow (conduction) welding mode. Several defects such as process instabilities (high fluctuations in the keyhole dynamics), formation, and collapse of the keyhole, as well as the lack of penetration, were detected from the corresponding signal signatures. In addition, this information permits us to locate the defect/transition site through the in situ and realtime quality control of the process in production lines. In contrast, porosity and cracks could not be sensed. (C) 2017 Laser Institute of America. [http://dx.doi.org/10.2351/1.4983507]
\end{abstract}

Key words: Laser process monitoring, real-time quality control, signal processing by wavelet analysis, optical sensor, light emission measurement

\section{INTRODUCTION}

Laser beam welding (LBW) has been widely used in manufacturing processes due to its high speed, noncontact, and precision with low heat effects. ${ }^{1,2}$ Upon increasing the use of lasers in material processing in industry, a primary concern over the industry spectrum is to detect defects fast, reliably, and cost-effectively. ${ }^{3}$ Therefore, next to the progress in laser material processing systems and welding strategies, ${ }^{4,5}$ the development of techniques for monitoring and online inspections are of particular importance to guarantee the quality of the products.

Optical detectors have been widely used over a wide range of industries since they are relatively simple, cheap, and effective. ${ }^{6,7}$ A number of quality monitoring systems based on one or more photodiodes have been reported by several authors. ${ }^{8-10}$ Although some commercial systems are available, signal processing and classification are crucial steps for further development of a real-time closed loop process control unit. ${ }^{11}$

The main objective of this contribution is to evaluate the potential of LBW process monitoring and defect diagnosis by using the optical sensors. To achieve this goal, the optical emission from the process zone was measured using optical sensors to monitor the weld quality of bead on Ti6Al4V (Ti Grade 5) plate with a single-mode Ytterbium fiber laser. Discrete wavelet transform (DWT) analysis was carried out to detect patterns and features that are not apparent in the raw signals. The intensity of the details (frequency components of the decomposed signal) has been considered to identify the characteristic frequencies used for the weld quality assessment. Consequently, the deep keyhole welding was confidentially distinguished from the conduction welding mode. Furthermore, several defects such as process instabilities (high fluctuations in the keyhole dynamics), formation, and collapse of the keyhole, as well as the lack of penetration, were detectable through their related signal signatures. Based on the present results, it is not only possible to identify some defects/transience of the process but also its location through the in situ and real-time quality control process that can be implemented in a production line. However, macrostructural defects such as the porosity and cracks were not traceable considering only the optical emissions.

\section{WAVELET ANALYSIS}

A simple and often used approach to process the recorded signals is to correlate the fast Fourier transform (FFT) 
spectrum with the laser welding quality parameters and defects. However, this method is principally limited to stationary and time invariant signals. ${ }^{12}$ In other words, this technique works for stable processes. In addition, FFT analysis does not retain the time domain information, while this would be a requisite to develop an in situ and real-time closed loop/ feedback control system. Hence, a time-frequency method is considered to be more appropriate even though it is a more sophisticated approach to analyze nonstationary signals of the laser material processing. ${ }^{12}$ Consequently, short-time Fourier transform (STFT) has been employed in several studies to analyze the process signals. ${ }^{13-15}$ Nevertheless, this method is intrinsically limited due to a resolution problem. ${ }^{12}$ Basically, STFT analyses the signal based on a fixed scale (width of the window/function). If the scale is chosen too small, then the large signals' details (i.e., larger than the width of the window) will not be detected properly (a good resolution in the time domain but a poor resolution in the frequency domain). On the other hand, with a larger window (scale), the details are detected well but the transform will not localize them (a good resolution in the frequency domain but poor resolution in the time domain). Therefore, there is a limit to the localization precision in time-frequency representation of a signal explained as the uncertainty principle in the textbooks. ${ }^{12,16}$ To solve the resolution problem, the transform had to be defined independent of the scale or with variable scales.

As an alternative approach, the WT was developed to overcome the resolution problem by introducing a family of modulation functions upon varying the scale. ${ }^{12,17}$ Accordingly, the WT is established based on a multiresolution analysis to provide a good time resolution for the high frequency components and a good frequency resolution for the low frequency components. ${ }^{17}$ This makes the WT an interesting approach to analyze signals (processes) which include high frequency components for short durations while low frequency components last longer. This multiresolution analysis enables a time-frequency representation of the signal to detect patterns/features that are not visible in the raw data. ${ }^{18}$ Compared with FFT, DWT not only decomposes the signal into different frequency bands through several sequential steps (i.e., decomposition levels) but also retains the time-domain information in the decomposed signal. This enables us to locate the position of the signal features and thus identify the defects locally.

According to the DWT principle,,${ }^{12,19}$ at each decomposition level, part of the frequency components (starting from the maximum acquired frequency) is extracted from the original signal in a sequential order. The extracted parts are socalled the details, whereas the remained signal is named approximation. The approximation signal can be further decomposed into a next level so that the signal is broken down into lower frequency components. ${ }^{12,17}$ According to the theory of wavelet decomposition, the signal after the $n$-th decomposition level can be expressed as follows:

$$
S=A_{n}+\sum_{i=1}^{n} D_{i}+\varepsilon
$$

where $S$ is the signal, $A$ and $D$ are the reconstructed approximation and details, respectively, while $\varepsilon$ represents the error which depends on the type of mother wavelet (modulation function) used to decompose the signal. The decomposition tree of the signal $S$ is illustrated in Fig. 1.

Among many existing wavelet families (such as Daubechies, Symlet, Coiflets, BiorSplines, Meyer, Gaussian, Mexican hat, and Morlet), in this work, Daubechies order 1, also known as the Haar wavelet was found to be the best mother wavelet that minimizes the error of the reconstructed signal as presented in Eq. (1). To process the signals, the three original signals acquired from the optical sensors are decomposed to level 12 by the $\mathrm{db} 1$ (Daubechies order 1 or Haar) wavelet.

\section{EXPERIMENTAL SETUP}

A single-mode fiber laser source StarFiber 150 P (Fiber laser-StarFiber $150 \mathrm{P} / 300 \mathrm{P}-$ long pulse fiber laser systems) with a $1070 \mathrm{~nm}$ wavelength was used in this work. The laser beam was transmitted through a single-mode optical fiber with a $12 \mu \mathrm{m}$ core diameter and focused at the surface of the sample by a focusing lens with a $170 \mathrm{~mm}$ focal length. This setup provides a spot size of about $30 \mu \mathrm{m}$ diameter at the focal point $\left(2 w_{0}\right)$. Co-axial back reflected and/or emitted light from the process zone (melt pool and its corresponding plume) is collected through the laser processing head. The collected light is directed toward a sensor module including three optical sensors (see Fig. 2).

Each sensor measures the light intensity at a particular waveband: Silicon sensor 450-850 nm, Germanium sensor $1000-1200 \mathrm{~nm}$, and InGaAs $1250-1700 \mathrm{~nm}$. Figure 3 depicts the spectral sensitivity range of the three optical sensors used in the present study. The output signals were fed into a data acquisition processor and recorded with a sampling rate of 10 $\mathrm{MS} / \mathrm{s}$, corresponding to a maximum frequency rate of $5 \mathrm{MHz}$.

The welding trials were performed on $2 \mathrm{~mm}$-thick plates of the Ti6Al4V alloy (Grade 5) due to the ease of the microstructural characterization of the different zones such as the fusion zone, heat affected zone, and base material.

A series of experiments have been carried out considering the effects of the laser peak power, pulse shaping, and ramping on the weld quality. However, in this contribution, we present and analyze only one specific experimental configuration that is shown in Fig. 4(c) and was repeated three times. The laser source was triggered by the positioning system (table). In other words, the table starts moving a couple of milliseconds in advance and is stopped after the laser illumination ends to prevent any potential overheating at both

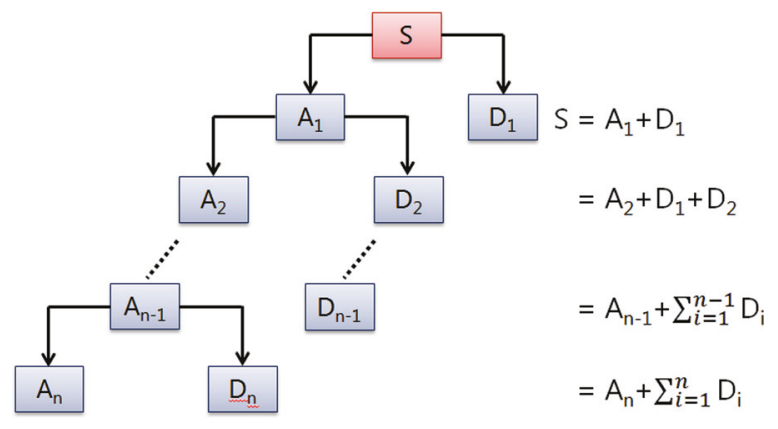

FIG. 1. Wavelet decomposition tree. 


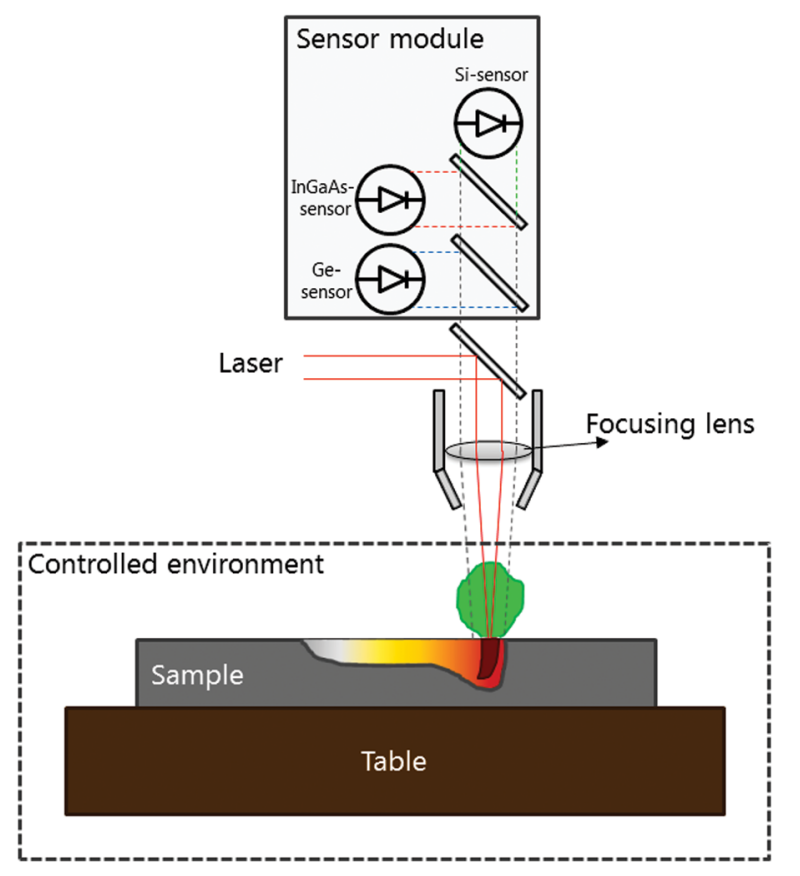

FIG. 2. Schematic overview of the experimental setup to monitor the laser welding process.

the starting and ending points. Accordingly, the moving sample receives the energy of the collimated laser beam to make a bead on plate seam weld (i.e., SHADOW welding as shown in Fig. 4(a)). As seen in Figs. 4(c) and 4(d), the laser illumination starts at a time of about $4 \mathrm{~ms}$ and lasts until $54 \mathrm{~ms}$ (i.e., the pulse width is $50 \mathrm{~ms}$ ). During the process, the sample is moved with a velocity of $100 \mathrm{~mm} / \mathrm{s}$. Thus, it produces a SHADOW weld of $5 \mathrm{~mm}$ length. In addition, the experiments were carried out in a controlled environment (e.g., a welding chamber filled with inert gas) to prevent any potential weld contamination such as oxidation.

\section{RESULTS AND DISCUSSION}

Figure 4 shows a typical laser weld together with its associated power and optical signals. The bead profile was observed using light microscopy from the top surface and the longitudinal cross section as shown in Figs. 4(a) and 4(b), respectively. From these figures, the weld width and penetration depth were measured. As expressed in Sec. III, a series of experiments have been carried out considering the effect of peak power, pulse shaping, and ramping on the weld quality. However, for the sake of brevity, only one sample of the experiment with pulse shaping is shown here. Thus, a single

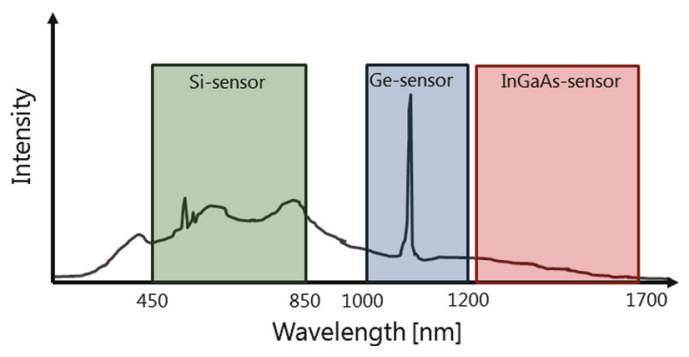

FIG. 3. Spectral sensitivity range of the three optical sensors.

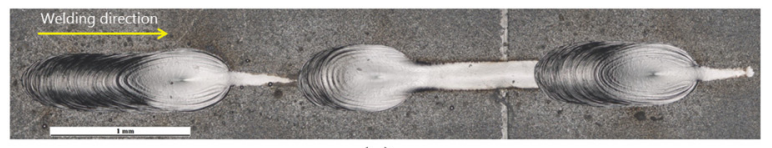

(a)

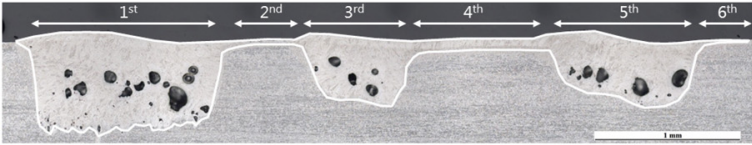

(b)

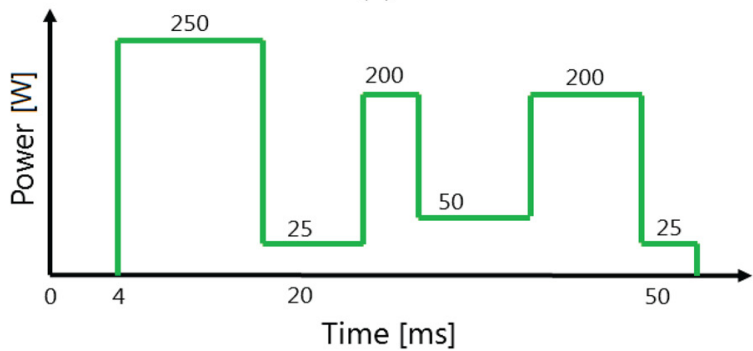

(c)

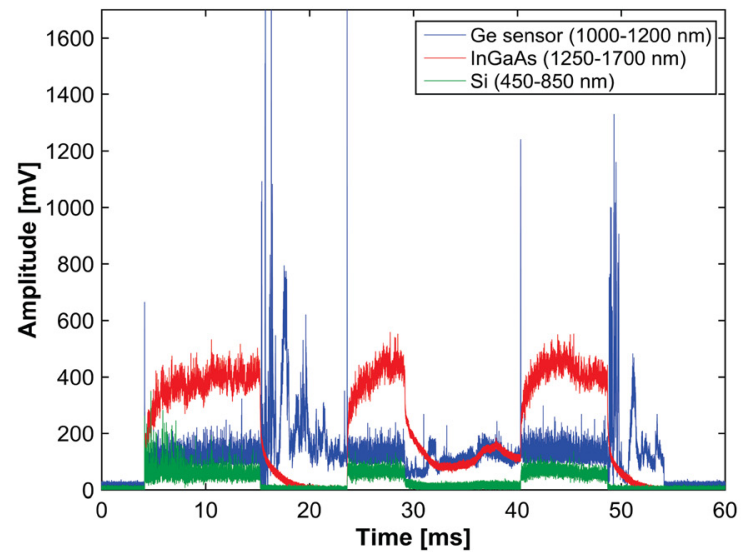

(d)

FIG. 4. Typical results of a modulated (pulse shaping) SHADOW welding on a Ti6Al4V alloy sample: (a) top surface, (b) longitudinal cross section, (c) the laser pulse shape, and (d) the acquired optical signals.

long pulse (a $50 \mathrm{~ms}$ pulse width) has been intentionally modulated several times to alter the power (or the energy density) as shown in Fig. 4(c). These laser parameters have been chosen based on a previous study. ${ }^{5}$ These parameters allow producing both shallow (conduction) and deep penetration (keyhole) welding modes within a single laser pulse. In addition, this prompts several welding defects such as porosity, material spattering, and lack of the fusion zone, and evidence of this is shown in Figs. 4(a) and 4(b). Through analyzing this example, we will be able to determine the limitation of each optical sensor by comparing the acquired signals in terms of their relative differences with respect to the corresponding signal features. Figure 4(d) plots the acquired optical signals utilizing the three available photodiode sensors.

Based on the visual inspection of the weld surface and cross section (shown in Figs. 4(a) and 4(b)), the weld line can be divided into six segments. Each segment corresponds to a prescribed power density extent and represents different welding modes/conditions. For instance, the first segment indicates a deep penetration (keyhole) welding mode with a relatively high level of porosity, which is potentially due to 
the instabilities in the process dynamics, in particular during the formation and collapse of the keyhole. The third and fifth segments are also considered as keyhole welding modes but with less penetration and porosity as compared to the first segment. The second and sixth segments display a very shallow depth penetration of only 10-20 $\mu \mathrm{m}$. We defined these two sections as the lack of penetration along the weld bead. In contrast, the fourth segment, with a penetration of about $100 \mu \mathrm{m}$ and an aspect ratio of 0.5 , is a typical representation of a shallow (conduction) welding mode.

We believe that each optical sensor provides some information about the welding process dynamics. The question remaining is which sensor provides which information of the welding process. Can keyhole and conduction welding be differentiated? Can the different defects be also distinguished? Which sensor is the most appropriate to complete this task? In this contribution, DWT is used to process the raw signals and to answer these questions.

\section{A. InGaAs and silicon sensors}

Since InGaAs (1250-1700 nm) and silicon (450-850 nm) sensors provided mostly similar information from the welding process, they are discussed together.

By comparing the bead profile with the optical signals in Fig. 4(d), one can investigate the correlation between the weld quality/defects (bead profile) and the optical signals. When comparing the laser power in Fig. 4(c) with the original signal, we clearly see the change between the keyhole and conduction welding mode. However, in the keyhole welding mode, the high amplitude is very much similar for the different laser powers despite producing noticeable differences in the penetration depth shown in Fig. 4(b). In addition, the difference between the lack of penetration and no laser illumination (before and after the laser pulse) is not clear. For instance, during the 2nd segment, between 16 and $24 \mathrm{~ms}$, there is almost no signal reflected back to the InGaAs and Silicon sensors although the material has been melted to a depth of $10-20 \mu \mathrm{m}$ and this is very similar to the sensor response recorded before and after the laser pulse (before $4 \mathrm{~ms}$ and after $54 \mathrm{~ms}$ ). These observations illustrate some limitations toward the direct analysis of the original signal. Hence, a deeper analysis by representing the signal in the time-frequency domain through the DWT is useful in order to extract the requisite information from the raw signals. Figures 5 and 6(b)-6(f) present the results of the DWT analysis and signal decomposition for the InGaAs and silicon (Si) sensors, respectively. The abscissa represents the time (in millisecond) while the ordinate is the signal amplitude (in $\mathrm{mV}$ ). To prevent redundancy, only the important information is shown in these figures for the further discussion.

These figures present the original signal, the approximate signal at level 12 (A12), as well as the detail signal at several decomposition levels (D6, D7, D11, and D12). The approximation signals (A12) are very similar to the original signals despite the fact that the high frequency components (beyond $1 \mathrm{kHz}$ ) have been extracted from the original signals. As a result, one can clearly see the changes in the approximation signal amplitude from keyhole welding to

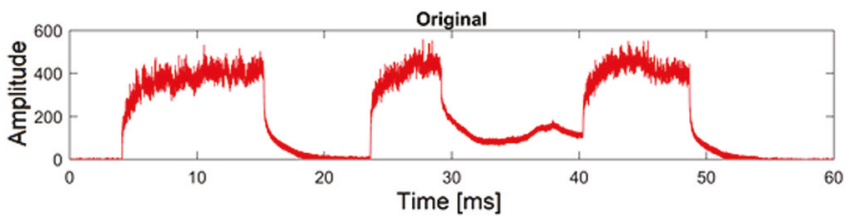

(a)

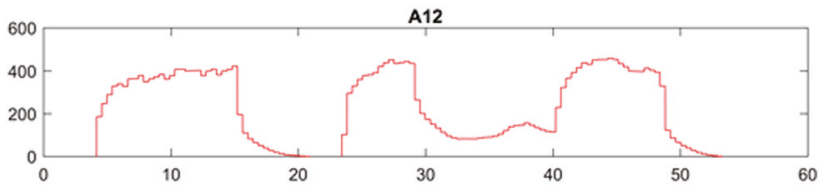

(b)

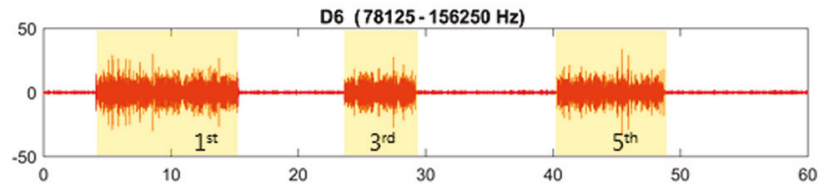

(c)

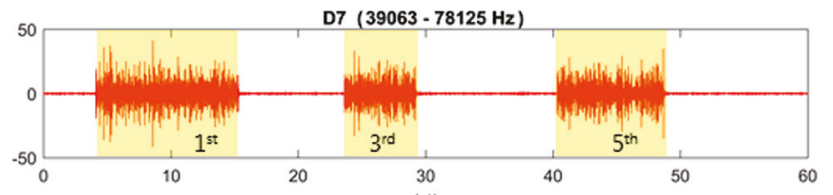

(d)

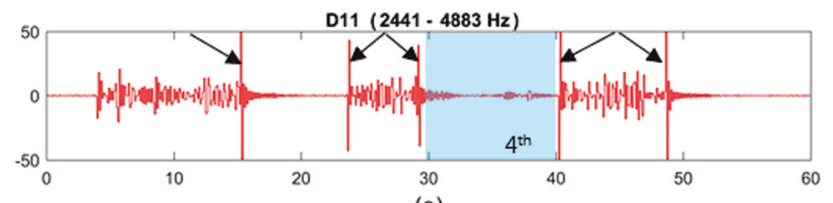

(e)

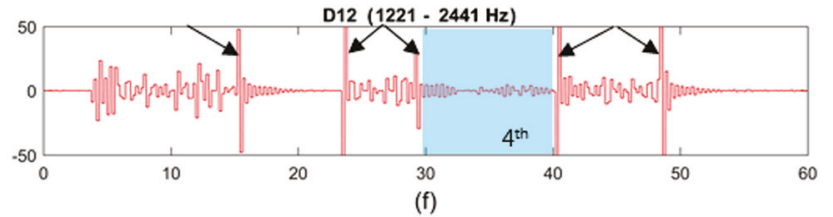

FIG. 5. Wavelet decomposition of the optical signal acquired by the InGaAs sensor: (a) original signal, (b) the level 12 approximate signal (A12), and the detail signal of (c) level 6 (D6), (d) level 7 (D7), (e) level 11 (D11), and (f) level 12 (D12).

conduction welding. Nevertheless, similar to what it was concluded with the original signal, the information related to the level of process dynamics, process instabilities, flow motion, material ejection, formation and collapse of the keyhole, penetration depth, and many other activities is also not apparent in the approximation signal. In fact, this information is extracted from the original signals and retained in the details of the signals through a sequential multilevel DWT. Each detail contains information of a certain frequency band.

Principally, the heat diffusion in the material is the most time-consuming phenomenon including the energy transformation and transmission during the laser processing. Therefore, it determines the rate of activities. However, the energy-coupling mechanism of the keyhole welding includes Fresnel absorption (i.e., absorption due to multiple reflections in the keyhole cavity) in addition to the surface absorption. In fact, the surface absorption is a dominant mechanism in conduction welding. ${ }^{20-22}$ Hence, the penetration of the light energy into the bulk material (i.e., faster energy transmission into the bulk material) along with the additional energy absorption (due to 


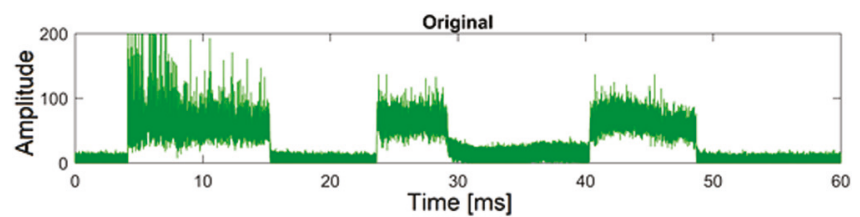

(a)

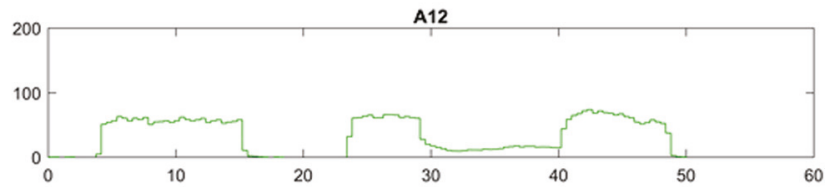

(b)

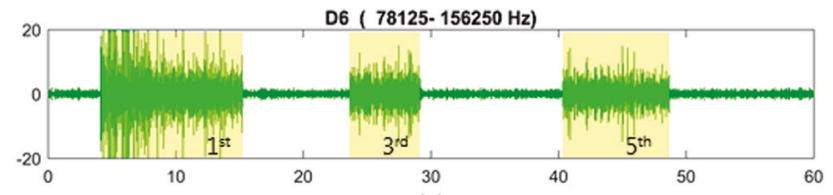

(c)

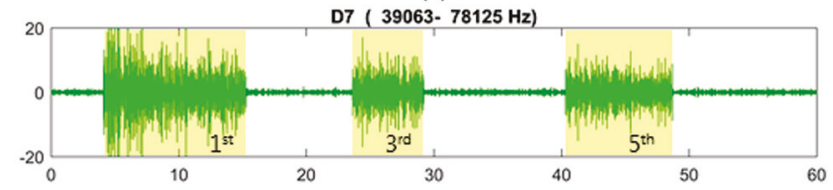

(d)

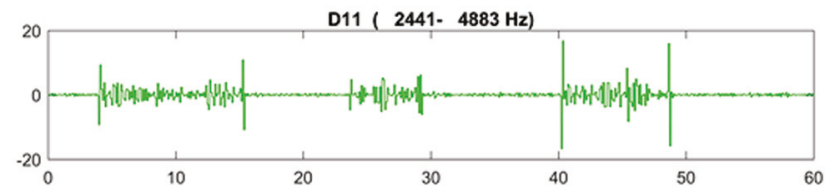

(e)

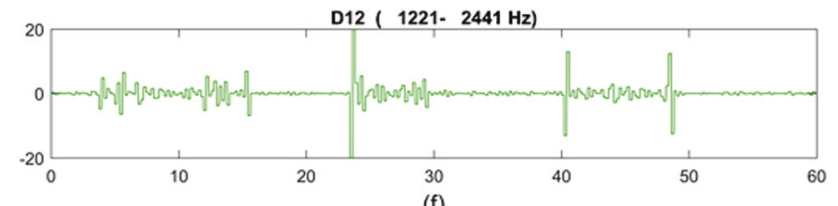

(f)

FIG. 6. Wavelet decomposition of the optical signal acquired by the Silicon sensor: (a) original signal, (b) the level 12 approximate signal (A12), and the detail signal of (c) level 6 (D6), (d) level 7 (D7), (e) level 11 (D11), and (f) level 12 (D12).

Fresnel absorption) in the keyhole welding leads to the promotion of the level of process dynamics (melting, evaporation, and flow motion) at relatively higher frequencies as compared to the conduction welding. Analyzing the results of DWT, we found that the keyhole welding activities are mostly characterized by the high frequency components $(40-150 \mathrm{kHz})$, whereas the conduction welding mode is represented by the lower frequency activities $(1-5 \mathrm{kHz})$. Consequently, the corresponding details of levels 6 and 7 together with those at levels 11 and 12 are used to obtain the desired information.

Scrutinizing the detail signals at decomposition levels 6 and 7 (D6 and D7, high frequency components) in Figs. 5, 6(c), and 6(d), we observe that the scatter and the amplitude of the details are much larger in the keyhole welding mode (1st, 3rd, and 5th segments highlighted in yellow) than in the conduction welding mode (2nd and 4th segments). This signal behavior is the key feature to distinguish between the keyhole and conduction welding behavior.

In addition, it is evident that the level of the scatter (e.g., the standard deviations of the signal coefficients or signal energy) in the 1st segment is larger than those in the 3rd and
5 th segments. Considering the results of the weld profile presented in Figs. 4(a) and 4(b), one can find out the correlation between the signal energy of the detail (D6 and D7 in Figs. 5 and 6) and the weld penetration depth at each segment. In other words, the signal energy of small segmentation (every $1 \mathrm{~ms}$ ) of the detail signal at high frequency components (D6 or D7) increases as the keyhole size becomes greater, thus indicating a deeper penetration depth.

Furthermore, the periodical formation and collapse of the keyhole bring some significant variations in the light emission intensity due to a change in either the temperature of the process zone or the size of the keyhole and its associated plume. Hence, the large process fluctuations or instabilities (such as keyhole oscillations, surface turbulence of the molten pool, and material ejection) induce relatively higher oscillations of the signal intensity. This is characterized by larger values of the amplitude in the detail signals as seen in Figs. 5, 6(c), and 6(d). This can be accounted as an indicator to measure the level of process instability. In other words, the number of large peaks in the detail signal measures the process instability (keyhole oscillation).

As explained earlier, the detail signals at decomposition levels 11 and 12 (D11 and D12, low frequency components) in Figs. 5 and 6 are representative of the long-term activities (in the range of about 0.2 to $1 \mathrm{~ms}$ ). Thus, they can be used as indicators to characterize the conduction/shallow welding mode. For instance, some oscillations in the detail D11 and D12 can be observed in the fourth segment (i.e., 30-40 ms highlighted in blue in Figs. 5(e) and 5(f)) known as the conduction welding mode which was not recognized at higher frequencies (D6 and D7). Similarly, the amplitude and signal energy of the corresponding detail signal (D11 and D12) can be used to measure the extent of conduction welding activities.

As indicated by the arrows in Figs. 5(e) and 5(f), very large peaks appeared within the detail signals D11 and D12. By comparing these peaks with the power variation steps seen in Fig. 4(c), one can conclude that these peaks are coincident with the changes in the power density level and thus with the keyhole formation and/or collapse during the welding process. This, in fact, manifests the heating and cooling rate at the beginning and the end of each segment.

\section{B. Germanium sensor}

Figure 7 presents the corresponding results for the Germanium sensor (1000-1200 nm), including the original data with level 12 of the approximate (A12) and four details (D6, D7, D11, and D12). By comparing the results for the three sensors, it is obvious that the signal obtained from the Germanium sensor is very different from that obtained from the other sensors.

Scrutinizing all signals obtained from this sensor (original, the approximate and details), it is found that this sensor is not ideal to determine the formation and collapse of the keyhole, its penetration depth, and/or process instabilities.

However, the most interesting information from this sensor is given by the detail decomposition levels 11 and 12 (D11 and D12, low frequency components) for the 2nd and 6th segments which are highlighted in red in Figs. 7(e) and 


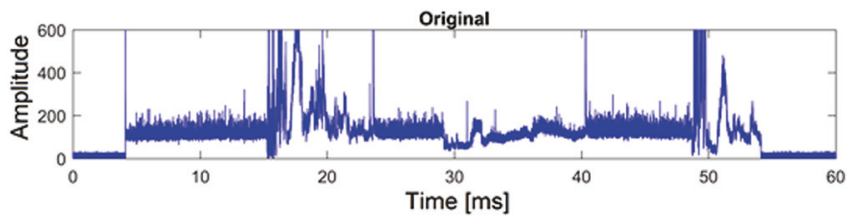

(a)

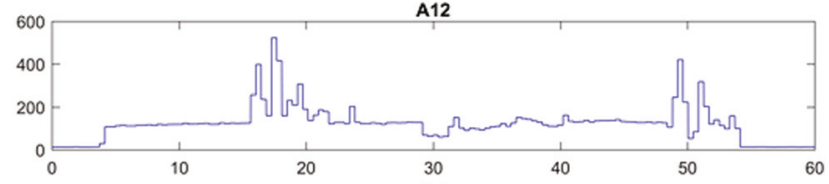

(b)

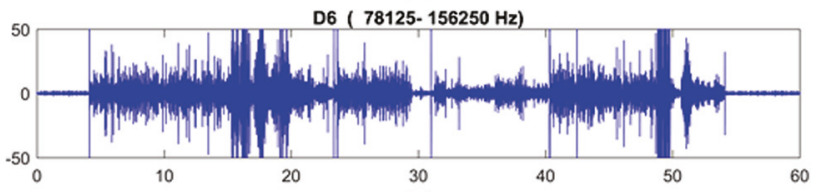

(c)

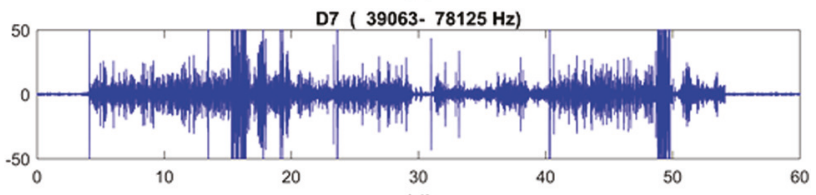

(d)

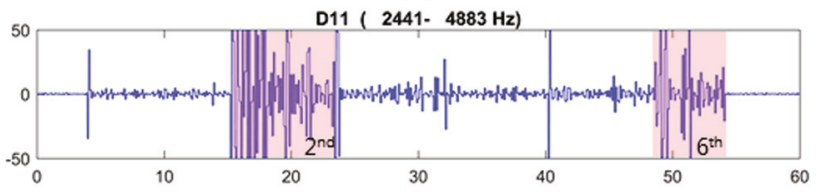

(e)

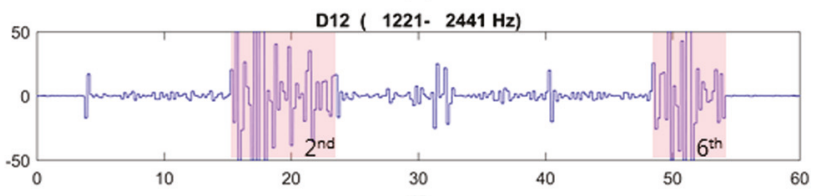

(f)

FIG. 7. Wavelet decomposition of the optical signal acquired by the Germanium sensor: (a) original signal, (b) the level 12 approximate signal (A12), and the detail signal of (c) level 6 (D6), (d) level 7 (D7), (e) level 11 (D11), and (f) level 12 (D12).

7(f). By comparing this figure with Fig. 4(b), we can conclude that the details of the signal from the Germanium sensor can be used to identify the lack of penetration in the weld bead. The high amplitude oscillations and scatters in the signal coefficients are good indicators to recognize the lack of the fusion zone. In fact, this high amplitude oscillation is originated from sensing a relatively high back reflected laser light (i.e., $1070 \mathrm{~nm}$ laser wavelength) which in turns implies the lack of (or less) light-material coupling, and thus is responsible for the lack of penetration. Therefore, the Germanium sensor and its corresponding signal are considered to be a very good candidate for examining the extent of laser light-material coupling.

\section{Summary of the results}

It is known that the material blow-out or spattering is expected to prompt some light emission changes. Nevertheless, there are several coincident process instabilities which also alter the light emission intensity. For instance, there are turbulences on the surface of the molten pool, surface ripples, plume transience, formation/collapse of the keyhole, keyhole oscillations, etc. Hence, no distinctive features can be found in the relevant signals to differentiate and localize these types of defects individually. However, as mentioned in Sec. IV A, the level of the process instability can be quantified by analyzing the detail signals of the InGaAs and/or Si sensor. Obviously, the structural defects, such as porosity formed within the bulk material, are also hardly detectable by these optical sensors.

With regard to these results, a statistical approach seems to be necessary to develop a statistical database. Such a database is of particular importance to be used as a benchmark in order to classify the weld quality according to the signal signatures. Consequently, it enables in situ and real-time control of the weld quality either by differentiating the heterogeneous welding modes or identifying the types of defects and their corresponding positions such as lack of penetration and process instabilities.

\section{CONCLUSIONS}

SHADOW laser welding experiments of $2 \mathrm{~mm}$-thick plates of the Ti6Al4V alloy (Grade 5) have been reported. The weld quality and defects have been considered in conjunction with the data from three optical sensors to investigate the possibility of having an in situ and real-time monitoring of the weld quality. The light emission signals of the recorded optical sensors were analyzed using the DWT. Compared to FFT, DWT not only decomposes the signal to different frequency bands but also retains the time-domain information in the decomposed signal. This enables us to locate the position of the signal features and thus may identify the defects locally.

Among the optical sensors used in the present study, the InGaAs and Silicon sensors were very effective in determining the heterogeneous types of welding processes (conduction versus keyhole welding) and their corresponding locations can be easily determined through the signal characteristics analysis. It was found that deep penetration mode (keyhole) welding leads to strong light emission intensity at high frequency bands $(40-150 \mathrm{kHz})$. The changes in the welding mode (deep to shallow penetration) declined the high frequency components to zero, whereas a small scatter in the signal was observed at lower frequency bands (1-5 $\mathrm{kHz}$ ). Such a behavior is a characteristic signature to represent the conduction welding mode. Also, these sensors gave a reliable evaluation of the penetration depth, the extent of the laser process dynamics, and process instabilities.

We found that, for the InGaAs and Silicon sensors, the amplitude of the high frequency components is proportional to the process dynamic level. Therefore, the number of large peaks in the detail signals measures the process instability (including keyhole oscillations, turbulence on the surface of the molten pool, material ejection, surface ripples, and plume transience). Furthermore, the standard deviation (or energy) of the detail signals at each segment disclosed an explicit correlation with the weld penetration depth.

In contrast, the Germanium sensor with a sensitivity range of 1000-1200 nm was found to be a very good candidate for evaluating the light-material coupling. In other 
words, it can detect the lack of penetration which was determined through the high amplitude oscillation in the corresponding detail signal.

Finally, although this analysis shows some promising results for in situ and real-time monitoring of SHADOW laser welding quality, it is not sufficient. To have a more robust method, a statistical method has to be used to classify the weld quality according to the signal signatures to develop a benchmark database for in situ and real-time assessment of the weld quality in the production line.

Nevertheless, the optical measurement techniques could not detect microstructural defects, such as porosity or cracks formed within the bulk material, since these types of defects are hardly detectable by the only use of the optical sensors. Therefore, additional detection techniques as well as the fusion sensing will be the next challenge for the authors to investigate.

${ }^{1}$ W. Steen and J. Mazumder, Laser Material Processing (Springer Science and Business Media, 2010).

${ }^{2}$ M. M. A. Khan, L. Romoli, and G. Dini, "Laser beam welding of dissimilar ferritic/martensitic stainless steels in a butt joint configuration," Opt. Laser Technol. 49, 125-136 (2013).

${ }^{3}$ H. Köhler, V. Jayaraman, D. Brosch, F. X. Hutter, and T. Seefeld, "A novel thermal sensor applied for laser materials processing," Phys. Proc. 41, 502-508 (2013).

${ }^{4}$ F. Vakili-Farahani, S. Favre, J. Lungershausen, and K. Wasmer, "Development and application of compact laser processing unit for gapbridging and bonding industries," in Proceedings of the 3rd International Conference on Nanojoining and Microjoining, Niagara Falls, ON, Canada, 2016.

${ }^{5}$ F. Vakili-Farahani, J. Lungershausen, and K. Wasmer, "Process parameter optimization for wobbling laser spot welding of Ti6Al4V alloy," Phys. Proc. 83, 483-493 (2016).

${ }^{6}$ A. Ancona and T. Sibillano, Monitoring Laser Welding (Woodhead Publishing Limited, Cambridge, England, 2008), pp. 260-287.

${ }^{7}$ T. Sibillano, A. Ancona, V. Berardi, and P. M. Lugarà, "A real-time spectroscopic sensor for monitoring laser welding processes," Sensors 9, 3376-3385 (2009).

${ }^{8}$ W. Gatzweiler, D. Maischner, and E. Beyer, "On-line plasma diagnostics for process-control in welding with CO2 lasers," in Proceedings of the 1988 International Congress on Optical Science and Engineering (1989), pp. 142-148.

${ }^{9}$ J. Fang, L. Li, Y. Chen, and L. Wu, "Wavelet analysis of plasma optical signals at pool penetration in laser welding," Proc. SPIE 5642, Information Optics and Photonics Technology, 403 (2005).

${ }^{10} \mathrm{~J}$. Tu, I. Miyamoto, and T. Inoue, "Characterizing keyhole plasma light emission and plasma plume scattering for monitoring $20 \mathrm{~kW}$ class CO2 laser welding processes," J. Laser Appl. 14, 146-153 (2002).
${ }^{11} \mathrm{~J}$. Shao and Y. Yan, "Review of techniques for on-line monitoring and inspection of laser welding," J. Phys.: Conf. Ser. 15, 101-107 (2005).

${ }^{12}$ J. Gomes and L. Velho, From Fourier Analysis to Wavelets (Springer, 2015), Vol. 3.

${ }^{13}$ S. Yang, W. Huang, D. Lin, F. Kong, and R. Kovacevic, "Monitoring of the spatter formation in laser welding of galvanized steels in lap joint configuration by the measurement of the acoustic emission," in Proceedings of the ASME 2008 International Manufacturing Science and Engineering Conference collocated with the 3rd JSME/ASME International Conference on Materials and Processing (2008), pp. 143-146.

${ }^{14} \mathrm{X}$. Gao, R. Wang, and Y. Yang, "Time-frequency characteristics clustering of metallic plume during high power disk laser welding," in Proceedings of the World Congress on Engineering and Computer Science (2012), pp. 24-26.

${ }^{15}$ A. S. Sun, E. Kannatey-Asibu, Jr., W. J. Williams, and M. Gartner, "Timefrequency analysis of laser weld signature," in Proceedings of the International Symposium on Optical Science and Technology (2001), pp. 103-114.

${ }^{16} \mathrm{~L}$. Cohen, "The uncertainty principle for the short-time Fourier transform and wavelet transform," in Wavelet Transforms and Time-Frequency Signal Analysis, edited by L. Debnath (Birkhäuser, Boston, MA, 2001), pp. 217-232.

${ }^{17} \mathrm{R}$. Polikar, The Wavelet Tutorial (Department of Electrical and Computer Engineering, Rowan University, 1996).

${ }^{18}$ R. Yan, R. X. Gao, and X. Chen, "Wavelets for fault diagnosis of rotary machines: A review with applications," Signal Process. 96, 1-15 (2014).

${ }^{19}$ D. P. Radunovic, Wavelets: From Math to Practice (Springer Publishing Company, Incorporated, 2009.

${ }^{20}$ M. Courtois, M. Carin, P. L. Masson, S. Gaied, and M. Balabane, "A new approach to compute multi-reflections of laser beam in a keyhole for heat transfer and fluid flow modelling in laser welding," J. Phys. D: Appl. Phys. 46, 505305 (2013).

${ }^{21}$ P. Berger and H. Hügel, "Fluid dynamic effects in keyhole welding-An attempt to characterize different regimes," Phys. Proc. 41, 216-224 (2013).

${ }^{22}$ S. Li, G. Chen, M. Zhang, Y. Zhou, and Y. Zhang, "Dynamic keyhole profile during high-power deep-penetration laser welding," J. Mater. Process. Technol. 214, 565-570 (2014).

\section{Meet the Author}

Dr. Farzad Vakili-Farahani majored in mechanical engineering at Ecole Polytechnique Federal de Lausanne (EPFL). $\mathrm{He}$ is currently serving as a post-doc in the laboratory of advanced material processing (LAMP) at Swiss federal laboratories for Materials science and technology (Empa). Since 2015, he has been working in the development of in situ and real-time monitoring systems and methods to control the quality of the laser material processing. Dr. Vakili is the coauthor of Encyclopedia of Two-Phase Heat Transfer and Flow. 